\title{
The challenges of organizing an international course in Latin America
}

\author{
Filippo Vairo, Mónica Luján López, Carolina Uribe Cruz, Priscila Gomes Corrêa and Guilherme Baldo \\ Centro de Terapia Gênica, Hospital de Clinicas de Porto Alegre, Porto Alegre, RS, Brazil.
}

\begin{abstract}
The Latin American School of Human and Medical Genetics (ELAG) is the main course of its kind in the genetics field in Latin America. Here we describe the main challenges regarding the organization of such event, including how we obtain funding and how we proceed with student selection. Thus, we aim to share our experience with other groups that intend to follow this format to create similar events in other areas in this region of the world.
\end{abstract}

Keywords: genetics in Latin America, funding, educational initiatives.

\section{Introduction}

The Latin American School of Human and Medical Genetics (ELAG) recently reached its $10^{\text {th }}$ anniversary and is the main course in its field on the continent. ELAG enrolled over 600 students from different Latin American countries over the past nine enrollments. Based on the feedback obtained from the students and faculty members, we believe that this type of initiative should be extended to other areas. Therefore, we aimed to enumerate the main challenges that we face every year while organizing this event. We hope that other groups can use the experience that we describe in this work and use it to create similar events for Latin American audiences.

\section{Challenges in Obtaining Funding}

The first and major challenge when organizing any event is obtaining funding, especially when the event is new. In Brazil, governmental agencies, such as Conselho Nacional de Desenvolvimento Cientifico e Tecnológico $(\mathrm{CNPq})$ and Coordenação de Aperfeiçoamento de Pessoal de Nível Superior (CAPES), have funding programs that provide support for conferences and courses. Additionally, international organizations, such as the United Nations University (UNU), have specific programs to support events and training programs in the Latin American region that involve Latin American countries and have supported most ELAG conferences.

Besides these options, additional and important sources of funding have come from private companies. Depending on the scientific field of the conference, companies may be interested in funding the event or even sponsoring a

Send correspondence to Guilherme Baldo. Centro de Terapia Gênica, Hospital de Clinicas de Porto Alegre, Ramiro Barcelos 2350, 90035-903 Porto Alegre, RS, Brazil. E-mail: guibaldo@ig.com.br. talk as an addendum to the main program. Both the company and the organizers should guarantee that the information included in the talk and the topic is relevant for the students. This type of support is mutually beneficial, allowing companies to provide new information while preserving an academic and scientific atmosphere in the main conference.

Finally, although each student receives a scholarship (provided with the funds received) that covers expenses related to transportation to the venue, accomodation and meals, approximately $30 \%$ of the total cost remains to be covered by a contribution from the students. This enables the organizing committee to provide adequate materials (binders, flash-drives, backpacks) for the conference. This contribution also serves as a confirmation of interest from the student, who should mobilize local supporters to allow his/her participation.

\section{Challenges in Selecting Students}

The main objective of the selection process is to receive the best representatives from as many Latin America countries as possible and to promote the communication and collaboration among scientists.

The selection process starts with the promotion of the event at ELAG's website and continues with an e-mail to faculty members, scientists and organizations all around Latin America. However, a more efficient advertisement comes from students who previously attended the School and discussed it with their colleagues.

The online registration process requests personal information and information about the academic background and curriculum vitae of applicants. Applicants are selected based on their academic level (most are MSc or PhD students) and professional profile (young MDs and health professionals are welcome). Applicants also provide a state- 
ment of interest for attending the School, which is critical for the selection process. Annually, approximately 150 students apply from the different countries of Latin America, but only 75 students are selected by the organizing committee. The selection criteria also attempt to represent as many Latin American countries as possible. Most of the students selected are Brazilian (approximately 50\%), which is not surprising because the conference is held in Brazil and because Brazil represents approximately $1 / 3$ of the Latin American population (nevertheless, we attempt to ensure that all regions of the country evenly represented). Both Argentina and Colombia have been historically well represented, but each year, ELAG has student attendees from approximately 15 Latin American countries.

The students who are not initially selected are placed on a waiting list and are called if the selected students do not confirm their participation. The reasons for selected students to not confirm participation is a lack of funds (although they receive a substantial fellowship, they still have to pay a portion of the costs and for the transportation to Porto Alegre).

All of the confirmed students receive a letter with instructions for payment and information about the event, the venue, the dress code, transportation and weather. Additionally, they receive information about hotels in the area in case they would like to extend their travel before or after the School. A member of ELAG's organization maintains direct contact with the selected students and during the conference provides assistance with arrivals, guidance to the hotel and handles any special situations.

\section{Organizational and Operational Challenges}

The organization of a School such as ELAG starts during the previous edition, where every strategy is evaluated, with the aim of improving the School for the next year. We attempt to hold the event in the same venue so that both the local team and the organizing team are familiar with the available activities so that we can provide the best experience for students and faculty members.

An important factor for students and faculty members is easy access to the venue, so it is important to hold the conference close to an international airport. We chose a hotel with adequate space and trained staff that could accommodate a delegation of approximately 120 people that was reasonably close to an urban area but relatively isolated, in the attempt to foster interaction among students and faculty members. During the School, students are encouraged to maintain contact with faculty during lunches, dinners, meetings, workshops and free time.

The scientific committee meets regularly to discuss the program to ensure that the most important topics related to human genetics (such as Evolution, Population Genetics, Medical Genetics, Genetic Therapies and Ethics) and their application to human health are covered. A group of faculty members consisting of renowned researchers from Latin America, North America and Europe is invited each year, with most coming back every year thereafter.

The organizing committee provides all of the necessary instructions and assists the selected students from the moment they are accepted. On the first day of the School, the committee welcomes students and faculty at the airport and helps them with bureaucratic issues related to language and culture as well as other needs besides transportation to the venue. During the School, students provide feedback on each activity and all of their opinions and suggestions are evaluated to improve future events.

\section{The Importance of a Genetics School in Latin America}

The Latin American countries are culturally and economically unique, and these differences are reflected in the access to information and to new technologies. With the organization of conferences such as ELAG, we try to reduce these differences, by offering a course that covers various areas of Human and Medical Genetics and provides opportunities for the discussion of projects and personal experiences.

The positive reviews that we receive each year along with the reports of the interactions between students and the faculty from different countries excites us. A number of collaborative projects born from interactions started at ELAG are already underway and there has been a substantial increase in the cooperation between different centers. The increased productivity resulting from such cooperation boosts the study of genetics in our continent, which is directly translated into new research related to diagnosis and therapy.

License information: This is an open-access article distributed under the terms of the Creative Commons Attribution License, which permits unrestricted use, distribution, and reproduction in any medium, provided the original work is properly cited. 